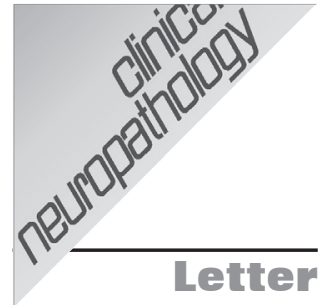

๑2018 Dustri-Verlag Dr. K. Feistle ISSN 0722-5091

DOI 10.5414/NP301093 e-pub: February 16, 2018
LETTERS TO THE EDITOR

\section{Calf muscle hypertrophy following S1 radiculopathy: A stress disorder caused by hyperactivity with variable response to treatment}

\author{
Nila Volpi ${ }^{1,2}$, Federica Ginanneschi ${ }^{1,2}$, \\ Alfonso Cerase ${ }^{1,3}$, Salvatore Francesco \\ Carbone $^{4}$, Margherita Aglianò ${ }^{1}$, \\ Paola Lorenzoni ${ }^{1}$, Matteo Bellini ${ }^{3}$, \\ Sabina Bartalini ${ }^{2}$, Giovanni Di Pietro ${ }^{5}$, \\ and Alessandro Rossi ${ }^{1,2}$
}

${ }^{1}$ Department of Medical, Surgical, and Neurological Sciences, University of Siena, ${ }^{2}$ Unit of Neurology and Clinical Neurophysiology, ${ }^{3}$ Unit of Neuroradiology, ${ }^{4}$ Unit of Diagnostic Imaging, and ${ }^{5}$ Unit of Neurosurgery, University Hospital Santa Maria alle Scotte, Siena, Italy

Sir, - Neurogenic focal muscle hypertrophy $(\mathrm{NMH})$ is a rare event in different instances of impaired nerve conduction: acquired and inherited polyneuropathies/multineuropathies [1, 2], post-polio syndromes [3], spinal stenosis, spinal muscle atrophies, radiculopathies [4]. Unilateral calf hypertrophy is an uncommon, but not unique, finding in S1 radiculopathy [5]. Muscle histology shows denervation, with atrophic as well as hypertrophic fibers, and frequent coexistence of inflammatory changes. Nevertheless, clinical presentation and responsiveness to treatment are not uniform. We report a 65 -yearold man, who presented with a 2-month history of painful progressive enlargement of the right calf, which started 5 months after an acute lumbago, causing pain of posterior right thigh, leg, and heel. The right calf was markedly enlarged $(7.5 \mathrm{~cm}$ right $>$ left), tense, and sensitive to touch/pressure, with no skin/subcutaneous changes, no involvement of the regional lymph nodes (Figure 1). Neurological examination detected weakness of right foot plantar flexion (3/5 MRC scale) and absence of right ankle jerk reflex; the patient complained of tingling paresthe- sias in the right foot and continuous calf pain. Routine blood tests, including sedimentation rate, C-reactive protein, eosinophil count, thyroid hormones, liver enzymes, and cryocrit were within normal ranges. ANA-ENA screening and search for neurotrophic viruses were negative. Serum creatine kinase (CK) was increased (520 UI/L).

Needle electromyography (EMG) showed marked abnormalities consisting of continuous complex repetitive discharges (CRDs), polyphasic motor unit potentials with reduced voluntary recruitment in the right gastrocnemius muscle. EMG recordings of right tibialis anterior, quadriceps femoris, gluteus maximum, and adductor magnus muscles were unremarkable.

Magnetic resonance imaging (MRI) (Figure 1) showed a volume increase of right leg posterior muscles, more evident on soleus and medial gastrocnemius, with intrafascial edema and mild hypervascularization of hypertrophic muscles.

Lumbosacral spinal MRI and computed tomography (Figure 1) evidenced diffuse degenerative disease of the lumbar spine and a L5-S1 right paramedian posterior disc herniation compressing the ipsilateral $\mathrm{S} 1$ radicular sheath and S1 nerve root.

A biopsy of the medial right gastrocnemius muscle (Figure 2) showed grossly hypertrophic fibers, grouped atrophy, and sparse myonecroses. Target lesions indicated denervation in most hypertrophic fibers. Inflammation consisted of scattered foci of mononuclear cells surrounding necrotic fibers. MHC-I (HLA-ABC) neolocalization was restricted to necrotic/regenerating fibers. Focal increase of acid phosphatase (AP) activity and cytoplasmic patchy deposits of complement terminal complex (membrane attack complex: MAC) were detected in the largest fibers with myofibrillar disarray. Immunohistochemistry for myosin heavy chains (MyHC) showed reactivity of all fibers for the slow isoform, with a milder stain in fibers coexpressing fast myosin, resulting in transitional hybrid fibers $(\sim 40 \%)$. Recurrent regenerating fibers coexpressed neonatal/fast/slow myosin. By morphometric analysis, the frequency peak of fiber equivalent diameters was observed at $\sim 100 \mu \mathrm{m}$, with recurrent elements being larger than $200 \mu \mathrm{m}$. 

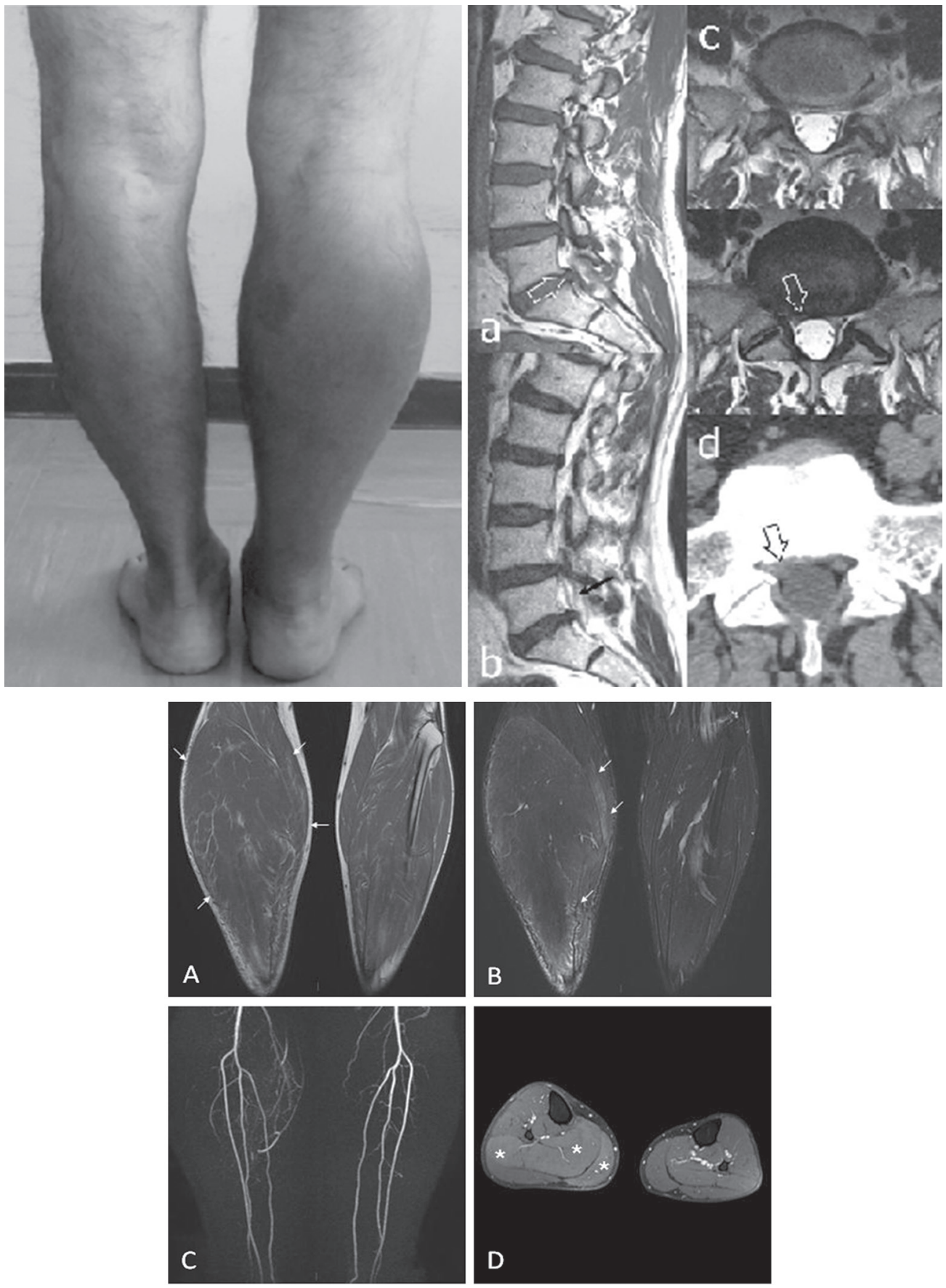

Figure 1. Top left: grossly hypertrophic right calf. Top right: lumbar spine neuroimaging. T1- (A) and T2weighted (B) sagittal, T2-weighted consecutive axial (C) MRI, and axial CT (D): disc and facet joints degenerative disease at right side of L5-S1 level, including zygapophyseal osteophytes (white closed arrow) and paramedian disc herniation (open arrows) in the right S1 lateral recess, compressing ipsilateral S1 root (black closed arrow). Bottom: MRI of the legs. T1-weighted image (A): volume increase of soleus and medial gastrocnemius muscles (white arrows). Fat-suppressed T2-weighted image: edema, with interfascial component (arrows). No fat involution was detectable by T1/T2 images. Regular popliteal artery run-off excluded vascular impairment (C). Steady-state post-contrast T1-weighted image (D): muscle masses showed homogeneous enhancement $\left({ }^{*}\right)$ because of increased interstitial spaces due to inflammatory components.

Steroid treatment was carried out with prednisone $50 \mathrm{mg} /$ day for 3 weeks, with no clinical remission. Consequently, the patient was submitted to CT-guided percutaneous transforaminal periganglionic, and trans- articular zygapophyseal infiltration of the right L5-S1 root with a mix of local anesthetic (Ropivacain, $2 \mathrm{mg}$ ) and corticosteroid (Triamcinolon, $40 \mathrm{mg}$ ), with considerable clinical remission: shortly after the proce- 

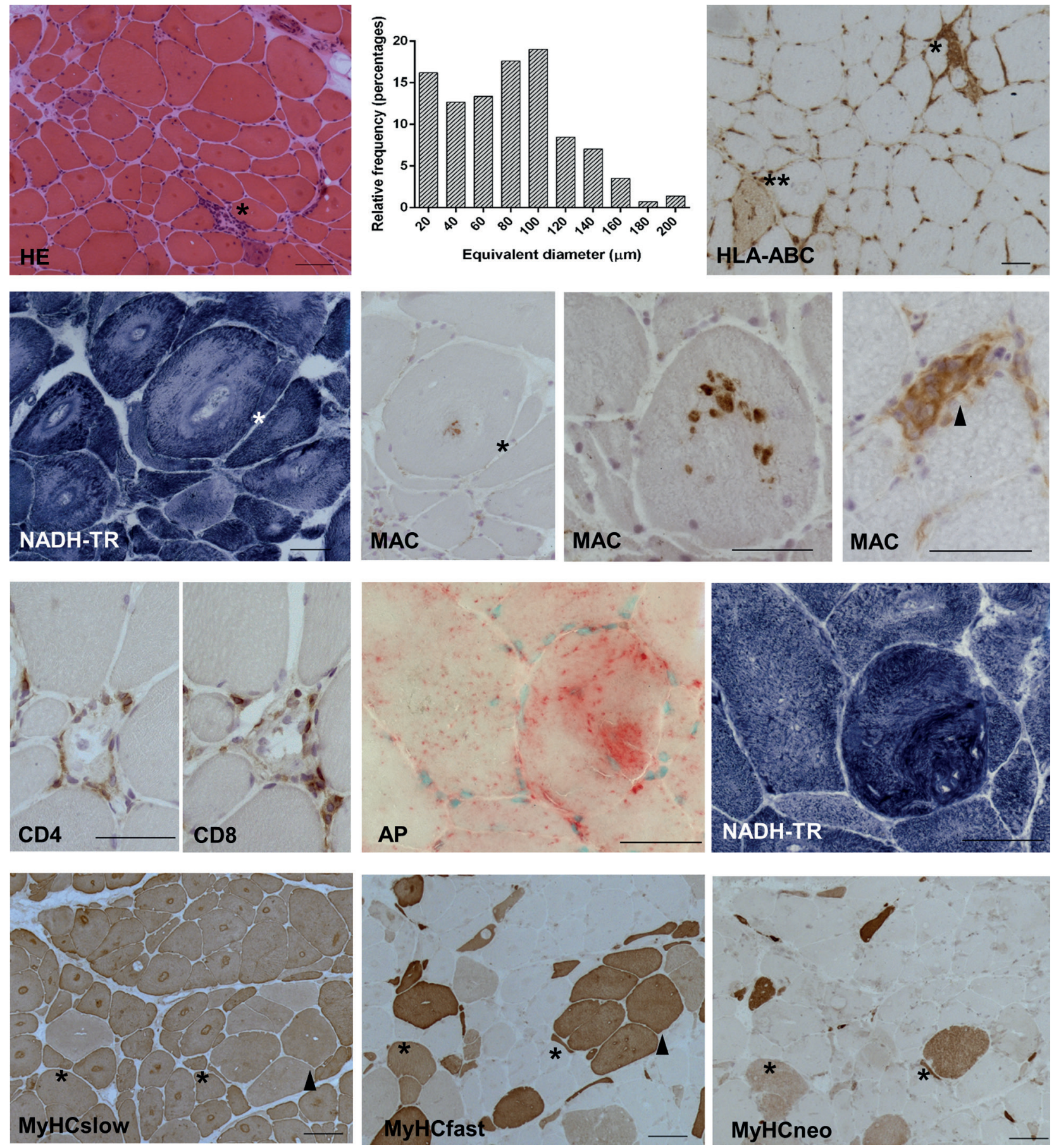

Figure 2. Medial right gastrocnemius muscle: biopsy findings. Hematoxylin-eosin: myonecrosis with mononuclear invasion $\left({ }^{*}\right)$; atrophic/hypertrophic fibers, internalized nuclei; target lesions as hypereosinophilic areas. Histogram of fiber equivalent diameter: high percentage of diameters $>100 \mu \mathrm{m} ;>15 \%$ small atrophic fibers. MHC-I (HLA-ABC) IP: only necrotic $\left(^{*}\right)$ and sparse degenerating/regenerating $\left({ }^{* *}\right)$ fibers show neolocalization. NADH-TR: target lesions as areas devoid of enzymatic activity with peripheral hyperreactive rim and coarse architectural changes. MAC IP: patchy cytoplasmic spots on target fibers (successive sections ${ }^{*}$ ), contrasting with uniform cytoplasmic stain in necrotic fibers invaded by mononuclear cells (arrowhead). CD4 - CD8 IP on successive sections: only necrotic fibers present with infiltrates. Acid phosphatase hyperactivity in a grossly hypertrophic fiber, with severe disruption of myofibrillar organization, as shown in successive section stained for NADH-TR. Serial MyHCs IP: all fibers express slow $\mathrm{MyHC}$, target lesions show a hyperreactive rim; variable intensity of MyHC slow stain in grouped fast fibers (arrowhead). Regenerating fibers $\left(^{*}\right)$ coexpress neonatal, slow, and fast isoforms. (NADH-TR = reduced nicotinamide adenine dinucleotide-tetrazolium reductase; AP = acid phosphatase; IP = immunoperoxidase; scale bar: $50 \mu \mathrm{m})$. 
dure, pain and muscle tenderness decreased and within 4 weeks a $2.5-\mathrm{cm}$ reduction of the right calf circumference was discernible. Nevertheless, 2 months later, pain and swelling recurred so that surgical discectomy was performed. Three months later, the right calf circumference, although still hypertrophied, was $3.5 \mathrm{~cm}$ less. Clinical examination showed full recovery of the right plantar flexion (MRC: 5), a sharp decrease of the foot paresthesia, and a reduction of the calf tension. EMG demonstrated a reduction of CRDs with improved muscular recruitment.

Coexisting inflammatory changes have led to inclusion of NMH into the domain of focal myositis (FM) [5, 6], a benign pseudotumor lesion within a single skeletal muscle, predominant at the lower limbs, possibly recurrent or evolving into diffuse myositis [7]. Unlike the present case, histology of pure FM usually shows no neurogenic changes and a prominent inflammation $[8,9]$.

In most calf NMH cases, increased CK plasma levels and focal inflammation likely represent a reactive response to degeneration remodeling of denervated fibers [5]. Correspondingly, MHC-I upregulation and T-cell deposits were extremely limited in our patient's gastrocnemius muscle, in contrast to the usual pattern of myositides. It is noteworthy that the pathophysiology of NMH does not seem to be univocal. In most radiculopathy-associated NMH cases, CRDs, generated by denervated fibers, are likely to act as hypertrophying agents, in analogy with increased muscle mass and fiber diameters obtained by functional muscle electrical stimulation, both experimentally [10] and in conus/cauda syndrome patients [11]. Nonetheless, NMH reports in absence of CRDs $[5,12]$ suggest coexisting pathomechanisms, such as compensatory overload; accordingly, in our case, a minority of hypertrophied "non-target" fibers, thus possibly not denervated, were detected.

The unusual cytoplasmic multifocal deposits of MAC on MHC-I-negative, hypertrophic fibers, might therefore depend on the double stress of denervation and hyperactivity, with subsequent increase of intracellular proteases and degenerative events, also consistent with focal AP reactivity. In our patient, steroid unresponsiveness was congruous with paucity of muscle inflammatory changes. However, the varying response to steroids does not strictly correlate with inflammation, neither in FM [9] nor in radiculopathy-associated NMH [6], suggesting, besides a direct action on radicular compression, a steroid activity on the suppression of the membrane excitability.

Genetic factors or selective axon vulnerability have been suggested to be responsible for the uncommon occurrence of large "atrophy-resistant" fibers in muscle of subjects with spinal cord injuries [13]; yet, unrecognized motoneuron/muscle molecular features might also explain the low prevalence of NMH associated with the relatively frequent $\mathrm{S} 1$ radiculopathy.

A transitional "hybrid" fiber phenotype, coexpressing different isoforms of myosin and other contractile proteins, has been reported in denervated muscle, with a shift to fast type 2 fibers [14], whereas a fast-to-slow shift occurs following electric stimulation or excessive exercise [15]. Our finding of an ongoing major transition to the slow phenotype suggests that histotype shift, from continuous muscle activity, might cause the reported type 1 predominance in gastrocnemius NMH [3]. Serial MyHC immunohistology for analysis of histotype transition was not, to our best knowledge, previously performed in NMH. Comparison of this feature in CRD+ and CRD- cases of neurogenic muscle hypertrophy would be of interest.

In our patient, with conspicuous repetitive discharges causing type shift and degenerative muscle changes, only surgery led to stable improvement. The case calls for attention to the relevance of a thorough instrumental and pathological investigation in the uncommon occurrence of NMH, with the aim of establishing the most effective therapeutic strategy.

\section{Funding}

None.

\section{Conflict of interest}

The authors declare no conflict of interest. 


\section{References}

[1] Pareyson D, Morandi L, Scaioli V, Marazzi R, Boiardi A, Sghirlanzoni A. Neurogenic muscle hypertrophy. Report of two cases. J Neurol. 1989; 236: 292-295. CrossRef PubMed

[2] Uncini A, Di Muzio A, Chiavaroli F, Gambi D. Sabatelli M, Archidiacono N, Antonacci R, Marzella $R$, Rocchi $M$. Hereditary motor and sensory neuropathy with calf hypertrophy is associated with 17p11.2 duplication. Ann Neurol. 1994; 35: 552-558. CrossRef PubMed

[3] Gutmann L. AAEM minimonograph \#46: neurogenic muscle hypertrophy. Muscle Nerve. 1996; 19: 811-818. CrossRef PubMed

[4] Rowin J, Meriggioli MN. Complex repetitive discharges: cause or effect of neurogenic muscle hypertrophy? Muscle Nerve. 1999; 22: 1603-1606. CrossRef PubMed

[5] Lunde HM, Skeie GO, Bertelsen AK, Karlsen B, Miletic H, Lindal S, Brautaset NJ, Bindoff LA. Focal myositis - neurogenic phenomenon? Neuromuscul Disord. 2012; 22: 350-354. CrossRef PubMed

[6] Hemmi S, Shirakawa S, Kurokawa K, Sunada Y. Unilateral calf hypertrophy and focal myositis induced by S1 radiculopathy: dramatic response to steroid treatment. BMJ Case Rep. 2013; 2013: bcr2013200870. CrossRef PubMed

[7] Flaisler F, Blin D, Asencio G, Lopez FM, Combe $B$. Focal myositis: a localized form of polymyositis? J Rheumatol. 1993; 20: 1414-1416. PubMed

[8] Auerbach A, Fanburg-Smith JC, Wang G, Rushing $E J$. Focal myositis: a clinicopathologic study of 115 cases of an intramuscular mass-like reactive process. Am J Surg Pathol. 2009; 33: 1016-1024. CrossRef PubMed

[9] Toti P, Romano L, Villanova M, Zazzi M, Luzi P. Focal myositis: a polymerase chain reaction analysis for a viral etiology. Hum Pathol. 1997; 28. 111-113. CrossRef PubMed

[10] Pette D, Vrbová G. The Contribution of Neuromuscular Stimulation in Elucidating Muscle Plasticity Revisited. Eur J Transl Myol. 2017; 27: 6368. CrossRef PubMed

[11] Kern H, Rossini K, Carraro U, Mayr W, Vogelauer $M$, Hoellwarth $U$, Hofer $C$. Muscle biopsies show that FES of denervated muscles reverses human muscle degeneration from permanent spinal motoneuron lesion. J Rehabil Res Dev. 2005; 42 (3 Suppl. 1): 43-53. PubMed

[12] Streichenberger N, Meyronet D, Fiere V, Pellissier $J F$, Petiot $P$. Focal myositis associated with S-1 radiculopathy: report of two cases. Muscle Nerve. 2004; 29: 443-446. CrossRef PubMed

[13] Biral D, Kern H, Adami N, Boncompagni S, Protasi $F$, Carraro $U$. Atrophy-resistant fibers in permanent peripheral denervation of human skeletal muscle. Neurol Res. 2008; 30: 137-144. CrossRef PubMed

[14] Burnham R, Martin T, Stein R, Bell G, MacLean I, Steadward $R$. Skeletal muscle fibre type transformation following spinal cord injury. Spinal Cord. 1997; 35: 86-91. CrossRef PubMed

[15] Gondin J, Brocca L, Bellinzona E, D'Antona G Maffiuletti NA, Miotti D, Pellegrino MA, Bottinelli $R$. Neuromuscular electrical stimulation training induces atypical adaptations of the hu- man skeletal muscle phenotype: a functional and proteomic analysis. J Appl Physiol (1985). 2011; 110: 433-450. CrossRef PubMed

Correspondence to

Nila Volpi, MD

Department of Medical

Surgical and Neurological Sciences

University of Siena

Viale Bracci 16

53100 Siena, Italy

nila.volpi@unisi.it 